03

\title{
О нелинейной динамике массивных частиц в смерчах
}

\author{
(С) Л.Х. Ингель \\ Научно-производственное объединение „Тайфрун“, \\ 249038 Обнинск, Россия \\ Институт физики атмосфреры им. А.М. Обухова РАН, \\ 119017 Москва, Россия \\ Межведомственный центр аналитических исследований при Президиуме РАН, \\ 117997 Москва, Россия \\ e-mail: lev.ingel@gmail.com
}

Поступило в Редакцию 9 ноября 2019 г.

В окончательной редакции 9 ноября 2019 г.

Принято к публикации 2 декабря 2019 г.

Аналитически исследовано движение инерционных частиц в интенсивных вихрях с вертикальной осью в поле силы тяжести. В этой задаче существен нелинейный характер гидродинамического сопротивления его зависимость от модуля скорости движения частицы относительно среды. Разные составляющие движения взаимодействуют между собой, поскольку каждая из них влияет на коэффициент сопротивления. Найден эффективный способ приближенного решения задачи. Установлен ряд общих закономерностей динамики частиц. В частности, характерен режим, в котором их тангенциальная скорость близка к скорости движения среды, в то время как радиальная скорость существенно меньше (она близка по порядку величины к среднему геометрическому тангенциальной скорости частицы и отличия последней от тангенциальной скорости среды).

Ключевые слова: интенсивные вихри, массивные частицы, перенос, аналитическая модель, нелинейное сопротивление, смерчи.

DOI: $10.21883 /$ JTF.2020.06.49274.359-19

\section{Введение}

В настоящей работе теоретически исследуется движение инерционных частиц (частиц из вещества много плотнее среды - капель воды, песчинок и т.п.) в интенсивном стационарном осесимметричном вихре с вертикальной осью в поле силы тяжести. Такая задача представляет интерес для ряда приложений. От распределения частиц в атмосферном вихре зависят его радиолокационные и оптические свойства, а также ветровой напор на сооружения. Наблюдения переноса вещества смерчами (торнадо) давно используются для изучения динамики этих вихрей [1-3]. В [1] много внимания уделяется геологической роли переноса вещества сильными ветрами. Сепарация в вихре частиц разных размеров и веса может играть определенную роль в разделении электрических зарядов и, следовательно, в электромагнитных проявлениях интенсивных вихрей. Кроме того, в [2] предложена модель динамики смерча (во многом качественная), в которой принципиальную роль играет накопление тяжелого вещества (прежде всего капель) во внешней области вихря. Следует также упомянуть оформленную в качестве изобретения идею воздействия на атмосферные вихри $[4,5]$, в которой существенную роль играет внесение в вихрь облака тяжелых частиц.

Теоретическому исследованию движения частиц в атмосферных вихрях посвящен ряд публикаций, в частности, [5-8]. При больших значениях числа Рейнольдса задача существенно усложняется из-за нелинейной зависимости гидродинамического сопротивления от ско- рости. Коэффициент сопротивления зависит от модуля скорости частицы относительно среды. Это означает наличие нелинейного взаимодействия между разными составляющими движения частицы, поскольку все они влияют на коэффициент сопротивления. В последней из упомянутых работ найдены некоторые аналитические закономерности горизонтального движения тяжелых частиц с учетом упомянутого взаимодействия. При этом рассмотрение ограничивалось ситуациями, когда центробежная сила много больше силы тяжести, так что последней пренебрегалось. Это существенно сужает область применимости упомянутых результатов: они применимы лишь для весьма интенсивных вихрей и в относительно небольших областях пространства, в которых вертикальной составляющей движения можно пренебречь. В настоящей работе это ограничение снято - рассматривается задача с учетом силы тяжести. Кроме того, рассматривается более широкая область значений числа Рейнольдса для частиц.

Как и в [8], рассматривается вертикально-однородный вихрь, в котором задано распределение тангенциальной скорости $V(r)$, где $r$ - расстояние от вертикальной оси вихря. Задача о движении частицы в поле центробежных сил в некоторых отношениях аналогична движению в поле силы тяжести - в силу известной эквивалентности сил инерции и тяготения. Это обстоятельство делает возможным использование в данной задаче обширного опыта исследований гравитационного оседания частиц в атмосфере (см., например, [9-12]). Но важное отличие заключается в том, что по мере радиального движения 
частицы меняется как центробежная сила (в отличие от ее аналога - неизменной силы тяжести), так и сила вязкого взаимодействия частицы с воздухом. Действительно, от расстояния до оси вращения зависят и скорость частицы, и фоновая тангенциальная скорость воздуха $V(r)$. Следовательно, от $r$, вообще говоря, зависит и разность этих скоростей, определяющая взаимодействие частицы с воздухом. Поэтому становится содержательной даже элементарная на первый взгляд задача о вязком квазистационарном движении частицы (получение аналога формулы Стокса, описывающей вязкое оседание частицы в поле силы тяжести).

\section{Постановка задачи}

Три проекции уравнения вязкого движения частицы в полярной системе координат можно записать в виде

$$
\begin{gathered}
\frac{d u}{d t}=\frac{v^{2}}{r}-c u \\
\frac{d v}{d t}=-\frac{u v}{r}-c[v-V(r)], \\
\frac{d w}{d t}=-g-c w .
\end{gathered}
$$

Здесь $t$ - время, $g$ - ускорение свободного падения; $u, v, w$ - радиальная, тангенциальная и вертикальная составляющие скорости частицы (вертикальная ось направлена против силы тяжести); $c$ - коэффициент сопротивления; расстояние $r$ от оси можно рассматривать как лагранжеву радиальную координату частицы:

$$
u=\frac{d r}{d t} .
$$

Распределение тангенциальной скорости в вихре $V(r)$, как уже упоминалось, считаем заданным (для определенности ограничиваемся случаями, когда $V$ и $v$ не отрицательны).

По сравнению с [8], система уравнений дополнена уравнением (3), поскольку учитывается сила тяжести. Смысл данной системы уравнений достаточно очевиден: горизонтальное ускорение частицы определяется алгебраической суммой сил инерции (использована стандартная форма записи ускорений в полярных координатах) и силы вязкого трения. Последняя, в свою очередь, в рассматриваемом приближении определяется значением коэффициента сопротивления и скоростью частицы относительно среды. Коэффициент сопротивления $c=\tau^{-1}$ : $\tau$ - время вязкой релаксации [10]. Уравнения динамики подробно выводятся и анализируются, например, в [12]. В случае частиц конечных размеров, помимо учтенных выше сил, строго говоря, существуют и другие [12] (архимедова, „подъемная сила“, „сила присоединенной массы“), но в рассматриваемом приближении, в котором частицы предполагаются малыми, форма их - сферической, дополнительный учет упомянутых малых сил на данном этапе исследования не имеет смысла.
Масштаб времени $\tau$ в случае выполнения закона Стокса для достаточно малых частиц сферической формы выражается через коэффициент молекулярной вязкости среды $\eta$, радиус частицы $R$ и ее плотность $\rho_{p}[10]$ :

$$
\tau_{S}=2 R^{2} \rho_{p} / 9 \eta, \quad c_{S}=1 / \tau_{S}
$$

Для частиц бо́льших размеров и массы коэффициент сопротивления $c$ и время $\tau$ зависят от значения числа Рейнольдса для частицы:

$$
c=c(\operatorname{Re}), \quad \operatorname{Re}=\frac{2 R \rho|\mathbf{v}|}{\eta}=\frac{2 R|\mathbf{v}|}{v}, \quad v=\frac{\eta}{\rho} .
$$

Здесь $\rho-$ плотность среды (воздуха), $v-$ коэффициент ее кинематической вязкости, $\mathbf{v}$ - вектор скорости частицы относительно среды: ${ }^{1}$

$$
|\mathbf{v}|=\left[u^{2}+(v-V)^{2}+w^{2}\right]^{1 / 2} .
$$

Для коэффициента сопротивления при средних и больших числах Рейнольдса известны эмпирические формулы, полученные в результате обобщения многочисленных экспериментальных данных. Например, формула Клячко-Мазина

$$
c=c_{S}\left(1+\frac{1}{6} \operatorname{Re}^{2 / 3}\right)
$$

в интервале $3<\operatorname{Re}<400$ приводит к силе сопротивления, отличающейся от экспериментальных данных не более чем на $2 \%$ [10]. Для интервала $10^{3}<\operatorname{Re}<3 \cdot 10^{5}$ рекомендуется формула [9]

$$
c=\frac{1}{60} \operatorname{Re} c_{S}=\frac{|\mathbf{v}|}{l},
$$

где масштаб длины

$$
l=\frac{20}{3} \frac{\rho_{p}}{\rho} R .
$$

Для капель воды радиусом $0.5 \mathrm{~mm}$ в воздухе $l \approx 3 \mathrm{~m}$.

При известных функциях $c(\mathrm{Re})$ и $V(r)$ система уравнений для неизвестных $u, v, w$ и $r$ замкнута. В начальный момент $t=0$ считаем заданной радиальную координату частицы $r=r_{0}$. Остальные начальные условия будут при необходимости уточняться ниже.

Поскольку центробежная сила всегда направлена к периферии, ограничиваемся здесь рассмотрением ситуаций, в которых радиальная координата частицы $r$ монотонно растет со временем, иными словами, $u \geq 0 .{ }^{2}$

\footnotetext{
${ }^{1}$ Вместо диаметра частицы в выражении для числа Рейнольдса можно, разумеется, использовать радиус. Поэтому, например, в [9] и [10] значения чисел Рейнольдса при прочих равных условиях различаются в 2 раза.

2 Тем самым из рассмотрения исключаются некоторые классы начальных условий и движений. Например, рассматриваемые в [5], где преимущественно изучается движение частиц, „входящих“ в вихрь.
} 
В этом случае существует взаимно-однозначное соответствие между переменными $r$ и $t$, так что всегда возможен переход от одной переменной к другой; $d / d t=(d r / d t) d / d r=u(d / d r)$, и уравнения $(1)-(3)$ можно представить в виде

$$
\begin{gathered}
\frac{1}{2} \frac{d\left(u^{2}\right)}{d r}=\frac{v^{2}}{r}-c u, \\
u\left(\frac{d v}{d r}+\frac{v}{r}\right)=-c[v-V(r)], \\
u \frac{d w}{d r}=-g-c w .
\end{gathered}
$$

Уравнение (12) ниже будет удобно рассматривать также в виде

$$
[v-V(r)]=-\frac{u}{c}\left(\frac{d v}{d r}+\frac{v}{r}\right)
$$

\section{Предварительные гипотезы}

Выполненный в [8] анализ движения частиц без учета силы тяжести выявил ряд закономерностей, позволивших существенно упростить задачу, в частности:

1) для радиального движения характерен квазистационарный режим, когда левые части уравнений (1) и (11) пренебрежимо малы, так что имеет место приближенный баланс центробежных сил и сопротивления. Это аналогично задаче об оседании частицы в поле силы тяжести после завершения периода релаксации;

2) скорость азимутального движения частицы относительно близка к азимутальной скорости среды;

3) радиальная скорость частицы много меньше тангенциальной, но много больше разности $|v-V|$ :

$$
|v-V| \ll|u| \ll|V| .
$$

Можно предположить, что аналогичные закономерности выполняются и в рассматриваемой ниже более общей задаче. Поэтому примем в качестве предварительных гипотез следующие допущения: левые части уравнений (11) и (13) малы по сравнению с большими силами $V^{2} / r$ и $g$ соответственно; $|v-V| \ll\left(u^{2}+w^{2}\right)^{1 / 2}$, $V$, так что

$$
|\mathbf{v}| \approx\left(u^{2}+w^{2}\right)^{1 / 2}
$$

\section{Некоторые общие следствия}

При указанных допущениях уравнения (11), (13) принимают вид

$$
\begin{gathered}
c u \approx \frac{V(r)^{2}}{r}, \\
c w \approx-g .
\end{gathered}
$$

Отсюда следует весьма общее соотношение между составляющими скорости частицы, не зависящее от закона сопротивления:

$$
\frac{u}{w}=\approx-\chi^{1 / 2}(r) ; \quad \chi(r) \equiv\left[\frac{V^{2}(r)}{g r}\right]^{2} .
$$

Наблюдение за этими составляющими скорости движения частицы несет информацию о радиальном распределении скорости в вихре:

$$
V(r) \approx\left[g r \frac{u(r)}{(-w(r))}\right]^{1 / 2}
$$

Исключая $c$ из (12), (17), легко получить следующее соотношение между горизонтальными составляющими скорости частицы:

$$
u \approx\left\{\frac{V[V(r)-v]}{1+\xi}\right\}^{1 / 2}, \quad \xi \equiv \frac{d v / d r}{v / r} \approx \frac{d V / d r}{V / r}
$$

При любых реальных профилях $V(r)$ величина $\xi$ по порядку абсолютной величины обычно не превышает единицы. В еще большей степени это относится к величине $|1+\xi|^{1 / 2}$. Например, при степенном законе спадания скорости в вихре $V \sim r^{-\beta}, \xi=-\beta$ (отметим, что при $\beta>1$ частица опережает фоновый поток, поскольку переносит угловой момент от центра к периферии: $1+\xi<0, V-v<0)$. Поэтому, согласно (21), радиальная скорость близка по порядку величины к среднему геометрическому $|V-v|$ и $V-$ много больше первой из этих величин, но много меньше второй.

Из (14), (17) и (21) нетрудно получить выражение для тангенциальной скорости частицы:

$$
\begin{gathered}
v \approx V\left[1-4 \pi^{2}(1+\xi)\left(\frac{\tau}{T}\right)^{2}\right], \\
\tau \equiv \frac{1}{c}, \quad T \equiv \frac{2 \pi r}{V(r)} .
\end{gathered}
$$

Из (14) также следует:

$$
\left|\frac{V-v}{u}\right|=\tau\left(\frac{d v}{d r}+\frac{v}{r}\right) \sim 2 \pi \frac{\tau}{T} .
$$

Отсюда видно, что условие выполнения используемых гипотез $|V-v| \ll V, u-$ достаточная малость отношения времени релаксации движения частицы $\tau$ к периоду оборота вихря $T$.

Отметим, что (21), (22а) и (22б) не зависят от закона сопротивления (ранее они были получены без учета силы тяжести и лишь для одного конкретного закона сопротивления [8]), так что эти результаты достаточно универсальны.

\section{Случай линейной зависимости коэффициента сопротивления от модуля скорости}

$$
\begin{aligned}
& \text { Из }(9),(16)-(18) \text { следует } \\
& u \approx(g l)^{1 / 2} \frac{\chi^{1 / 2}(r)}{(1+\chi(r))^{1 / 4}}, \quad w \approx-(g l)^{1 / 2} \frac{1}{(1+\chi(r))^{1 / 4}}, \\
& c \approx\left(\frac{g}{l}\right)^{1 / 2}(1+\chi(r))^{1 / 4} .
\end{aligned}
$$


Из (23) и (4) можно выразить в квадратурах закон радиального движения:

$$
(g l)^{1 / 2} t \approx \int_{r_{0}}^{r} \frac{\left[1+\chi\left(r^{\prime}\right)\right]^{1 / 4}}{\chi^{1 / 2}\left(r^{\prime}\right)} d r^{\prime} .
$$

Зная $r(t)$, с учетом (19) нетрудно выразить и закон вертикального движения. Радиальное распределение скорости течения в вихре $V(r)$ может быть рассчитано по данным наблюдений за любой из составляющих скорости, например:

$$
V(r) \approx\left\{g r\left[\frac{(g l)^{2}}{w^{4}(r)}-1\right]\right\}^{1 / 2}
$$

При степенной зависимости $V(r)$ интеграл в (24), в принципе, вычисляется аналитически (полезно использовать интегрированную систему компьютерной математики „Mathematica“). Но в общем случае это приводит к весьма громоздким выражениям, трудным для анализа законов движения. В простейшем случае корневой зависимости $V(r)=V_{0}\left(r / r_{0}\right)^{1 / 2}, \chi=\left(V_{0}^{2} / g r_{0}\right)^{2}=$ const, так что выражения (23) не зависят от радиуса и времени, и частица движется с постоянной радиальной и вертикальной скоростью. Из (23) и (14) нетрудно убедиться, что используемое в настоящей работе предположение о малости величин $|(v-V) / V|,|(v-V) / u|$ выполняется при условии

$$
l / r \ll 1 .
$$

Если, например, характерный горизонтальный масштаб вихря порядка $30 \mathrm{~m}$, то значение $l$ должно быть меньше или порядка $3 \mathrm{~m}$, чему соответствуют капли воды размерами менее $1 \mathrm{~mm}$.

Из выражений (23) видно наличие нелинейного взаимодействия между радиальным и вертикальным составляющими движения частицы. Увеличение центробежной силы (значения $\chi$ ) ведет к росту радиальной скорости и коэффициента сопротивления, так что скорость оседания частицы при прочих равных условиях уменьшается. Если $\chi \gg 1$ (центробежная сила много больше силы тяжести), то

$$
\begin{gathered}
u \approx(g l)^{1 / 2} \chi^{1 / 4}=\left(\frac{l}{r}\right)^{1 / 2} V(r), \\
t \approx \frac{1}{\sqrt{l}} \int_{r_{0}}^{r} \frac{\sqrt{r^{\prime}} d r^{\prime}}{V\left(r^{\prime}\right)} \\
w \approx-\frac{(g l)^{1 / 2}}{\chi^{1 / 4}}=-\frac{g(l r)^{1 / 2}}{V(r)} \\
c \approx \frac{V(r)}{(l r)^{1 / 2}} .
\end{gathered}
$$

Это согласуется с предельным случаем, рассмотренным в [8], но теперь получено выражение и для вертикальной скорости, которая существенно ограничивается вследствие роста коэффициента сопротивления, обусловленного радиальным движением частицы. Нетрудно убедиться, что допущения, использованные при выводе (27), при достаточно гладких (например, степенных) зависимостях $V(r)$ справедливы при выполнении условия (26).

В противоположном предельном случае $\chi \ll 1$

$$
\begin{gathered}
u \approx(g l)^{1 / 2} \chi^{1 / 2}=\left(\frac{l}{g}\right)^{1 / 2} \frac{V^{2}(r)}{r}, \\
t \approx\left(\frac{g}{l}\right)^{1 / 2} \int_{r_{0}}^{r} \frac{r^{\prime} d r^{\prime}}{V^{2}\left(r^{\prime}\right)}, \quad w \approx-(g l)^{1 / 2}, \quad c \approx\left(\frac{g}{l}\right)^{1 / 2} .
\end{gathered}
$$

В частности, в области твердотельного вращения $V(r)=\Omega r$ получаем

$$
\begin{gathered}
r \approx r_{0} \exp \left(\Omega^{2} \sqrt{l / g} t\right), \quad u \approx \Omega^{2} \sqrt{l / g} r_{0} \exp \left(\Omega^{2} \sqrt{l / g} t\right), \\
v \approx V(r)=\Omega r_{0} \exp \left(\Omega^{2} \sqrt{l / g} t\right) .
\end{gathered}
$$

В этом предельном случае условия применимости приближенного решения еще более широки, чем (26). Проиллюстрируем это, оценив предполагавшееся малым отношение $|(v-V) / u|$. Согласно (14):

$$
\begin{aligned}
\left|\frac{v-V(r)}{u}\right| & =\left|\frac{1}{c}\left(\frac{d v}{d r}+\frac{v}{r}\right)\right| \sim\left(\frac{l}{g}\right)^{1 / 2}\left|\frac{V}{r}\right| \\
& \sim\left(\frac{l}{r}\right)^{1 / 2} \chi^{1 / 4} \ll\left(\frac{l}{r}\right)^{1 / 2} .
\end{aligned}
$$

Для капель воды радиусом $0.5 \mathrm{~mm}$ масштаб скорости $(g l)^{1 / 2}$ порядка $5 \mathrm{~m} / \mathrm{s}$, число Рейнольдса порядка $10^{3}$. При $\Omega=1 \mathrm{~s}^{-1}$ период вращения вихря около $6 \mathrm{~s}$, характерный временной масштаб в показателях экспонент (28) порядка $2 \mathrm{~s}$.

При бо́льших размерах частиц коэффициент сопротивления уменьшается, и, как видно из (22), это может существенно лимитировать пределы применимости использованного условия $|V-v| \ll V$.

\section{Анализ для закона сопротивления (8)}

При значениях числа Рейнольдса, больших нескольких десятков, формулу Клячко-Мазина (8) можно приближенно записать в виде $c \approx c_{S} \mathrm{Re}^{2 / 3} / 6$. В этом случае из (6) (16)-(18) следует

$$
\begin{gathered}
u \approx \chi^{1 / 2}(r) w \approx U \frac{\chi^{1 / 2}(r)}{(1+\chi(r))^{1 / 5}}, \\
w \approx-\frac{u}{\chi^{1 / 2}}, \quad c \approx \frac{g \chi^{1 / 2}}{u},
\end{gathered}
$$

где масштаб скорости

$$
U=\left[\frac{(2 R)^{4}}{v}\left(\frac{1}{3} g \frac{\rho_{p}}{\rho}\right)^{3}\right]^{1 / 5} .
$$


Результат наиболее сильно зависит от размеров частиц. Для капель воды радиусом $0.3 \mathrm{~mm}$ этот масштаб скорости порядка $3 \mathrm{~m} / \mathrm{s}$, число Рейнольдса порядка $10^{2}$, что и соответствует области применимости закона сопротивления (8).

Выше при постановке задачи не были сформулированы начальные условия для составляющих скорости $u, v, w$. Для первой и третьей они не требуются, поскольку рассматривается квазистационарный режим (предполагаются малые времена релаксации радиального и вертикального движения). Для тангенциальной скорости фактически предполагается, что начальные условия согласованы с найденными решениями (в противном случае возникает задача об адаптации к этим решениям, которая выходит за рамки настоящей работы).

\section{Заключение}

В работе найден эффективный способ приближенного решения нелинейной задачи, учитывающей зависимость сопротивления от скорости движения частицы относительно среды. Установлены некоторые существенные закономерности движений частиц в смерчах. Решения описывают, в частности, нелинейное взаимодействие разных составляющих движения, поскольку коэффициент сопротивления зависит от числа Рейнольдса, т.е. от всех составляющих скорости частицы. Результаты могут быть полезны и для решения обратной задачи получение информации о поле скорости в вихре по данным наблюдений о движении частиц. Найденные приближенные решения применимы до значений числа Рейнольдса порядка $10^{3}$ (для капель воды размерами до $1 \mathrm{~mm}$ ). Случай более массивных частиц с меньшими коэффициентами сопротивления также, несомненно, представляет интерес, но требует специального исследования.

\section{Конфликт интересов}

Автор заявляет, что у него нет конфликта интересов.

\section{Список литературы}

[1] Наливкин Д.В. Смерчи. М.: Наука, 1984. 110 с.

[2] Кушии В.В., Смерч. М.: Энергоатомиздат, 1993. 128 с.

[3] Природные опасности России. Т. 5. Гидрометеорологические опасности / Под ред. Г.С. Голицына, А.А. Васильева. М.: Крук, 2001. 296 с.

[4] Плешанов А.С. Патент РФ № 2027344. Способ подавления атмосферного вихря. Б.И. 2005. № 35.

[5] Плешанов А.С. К теории гидродинамической устойчивости смерчей (торнадо). М.: Информэнерго, 1993. 63 с.

[6] Островский Л.А. // Изв. АН СССР. Физика атмосферы и океана. 1990. Т. 26. № 12. С. 1307-1314.
[7] Лебедева Н.А., Осипцов А.Н. // Изв. РАН. МЖГ. 2009. № 1. C. 83-96. [Lebedeva N.A., Osiptsov A.N. // Fluid Dyn. 2009. Vol. 44. N 1. P. $68-79$.] DOI: $10.1134 / \mathrm{S} 0015462809010074$

[8] Ингель Л.X. // Изв. РАН. Физика атмосферы и океана. 2017. T. 53. № 4. C. 470-476. [Ingel L.Kh. // Izvestiya. Atmospheric and Oceanic Physics. 2017. Vol. 53. N 4. P. 413-418.] DOI: $10.1134 / \mathrm{S} 0001433817040065$

[9] Волощук В.М. Введение в гидродинамику грубодисперсных аэрозолей. Л.: Гидрометеоиздат, 1971. 208 с.

[10] Матвеев Л.Т. Курс общей метеорологии. Физика атмосферы. Л.: Гидрометеоиздат, 1984. 751 с.

[11] Ингель Л.Х. // ЖТФ. 2012. Т. 82. Вып. 11. С. 122-125. [Ingel L.Kh. // Tech. Phys. 2012. Vol. 57. N 11. P. $1585-1588$.] DOI: $10.1134 / \mathrm{S} 1063784212110126$

[12] Волков К.Н., Емельянов В.Н. Течения газа с частицами. М.: Физматлит, 2008. 600 с. 